\title{
GRANULOMATOSIS WITH POLYANGIITIS: A CASE REPORT
}

\begin{abstract}
Allena Prem Kumar1 ${ }^{1}$, Namballa Usharani², Gayatri Devi Yellapu³, Gorantla Samba Siva Rao ${ }^{4}$, Banavath Durga Prasad Naik ${ }^{5}$
\end{abstract}

\section{HOW TO CITE THIS ARTICLE:}

Allena Prem Kumar, Namballa Usharani, Gayatri Devi Yellapu, Gorantla Samba Siva Rao, Banavath Durga Prasad Naik. "Granulomatosis with Polyangiitis: A Case Report". Journal of Evolution of Medical and Dental Sciences 2015; Vol. 4, Issue 31, April 16; Page: 5397-5402, DOI: 10.14260/jemds/2015/789

\begin{abstract}
INTRODUCTION: Granulomatosis with polyangiitis (GPA) is an important form of AAV (ANCA associated vasculitis) syndrome. Being a multisystem disease, it can manifest in several combinations and can mimic an infection or malignancy. CASE PRESENTATION: A 55 years old man presented with cough, expectoration, haemoptysis, chronic otorrhoea and hearing loss. Imaging study suggested malignant mass lesion in right upper lobe with metastasis. HRCT of Temporal bones showed chronic sclerosing mastoiditis with bilateral CSOM. USG Abdomen and urine examination suggested acute glomerulonephritis. Trucut biopsy of lung showed granuloma and ANCA assays showed him C ANCA positive. Treatment with prednisolone and cyclophosphamide resulted in rapid resolution of symptoms and radiological clearance. CONCLUSION: Since GPA can effect almost any organ and a high degree of suspicion should be maintained in any multisystemic disease. Rapid work up with biopsy and ANCA Assay and early treatment with immunosuppressants will prevent irreversible organ damage.
\end{abstract}

KEYWORDS: GPA (Granulomatosis palyangiitis), ANCA (Anti Neutrophilic Cytoplasmic Antibodies), chronic sclerosing mastoiditis.

INTRODUCTION: Granulomatosis with polyangiitis (GPA) is an important form of ANCA associated vasculitis (AAV) syndrome. It is a multisystem disease and can affect almost any organ. Rapid diagnosis and prompt treatment are essential in order to minimise irreversible organ damage. We present a case of GPA who presented with right upper lobe mass like opacity on chest X ray and otological manifestations.

CASE PRESENTATION: A 55 years old male patient presented with complaints of productive cough with frequent small bouts of haemoptysis and lowgrade fever for the last 6 months. He also had symptoms of chronic discharge per both ears and mild hearing impairment for the last 2years. His past history revealed that he had full course of ATT with no relief. He was also treated for the CSOM for frequent exacerbation.

On examination he was febrile and tachypneic. He had no clubbing or palpable lymphadenopathy. Examination of respiratory system revealed bronchial breath sounds in right suprascapular and upper interscapular area and coarse crackles scattered over both sides of chest.

The blood investigation showed leukocytosis with elevated ESR (55 mm 1st $\mathrm{hr}$ ) and CRP $(32 \mathrm{mg} / \mathrm{dl})$. His serum creatinine was elevated $(2.1 \mathrm{mg} / \mathrm{dl})$. Urine examination revealed microscopic haematuria, proteinuria and presence of leucocytes. Subsequent cultures of urine did not show any growth.

Chest $\mathrm{x}$ ray showed right upper lobe mass like opacity with multiple nodular opacities in mid and lower zones. 
A CECT thorax followed and it showed a large soft tissue density equivalent mass measuring $8.6 \times 7.2 \times 11.2 \mathrm{~cm}$ in the right upper lobe, crossing the fissures and extending into lower lobes and encircling the upper lobe bronchus. Multiple nodular lesions were seen in both lungs, somewere cavitating and somewere surrounded by groundglass haziness. There was mediastinal lymphadenopathy and a small left pleural effusion. The features were suggestive of bronchogenic carcinoma with metastasis.

Ultrasound abdomen showed enlarged kidneys $(12 \mathrm{~cm} \times 12.7 \mathrm{~cm})$ and increased parenchymal echogenicity suggesting acute renal parenchymal disease.

ENT examination with otoscopy revealed bilateral central perforations. An audiogram was done and it confirmed bilateral moderate hearing loss. CT scan of temporal bones showed bilateral chronic sclerosing mastoiditis and bilateral chronic CSOM. There was ethmoidal sinusitis and deformed right tympanic membrane.

Flexible fiber optic bronchoscopy showed narrowing of right bronchus and no visible endobronchial abnormality. Bronchial washings were negative for AFB, fungi, and malignant cells.

Due to the combined pulmonary and renal manifestations, Granulomatosis with PolyAngiitis (GPA) with otic involvement was suspected.

Further investigation with CT guided trucut biopsy showed granulomas with moderate lymphocytic infiltrates, plasma cells and occasional gaint cells with fungal elements. There was no evidence of malignancy. Since fungal infection can coexist with GPA, further investigation for serum ANCA by immunofluorescence revealed C ANCA positive and P ANCA negative.

A diagnosis of GPA with otic involvement was made and the patient was initiated on prednisolone (1mg / kg wt), cyclophosphamide (2mg/kg wt) and cotrimoxazole 160/800 mg twice daily. In two weeks there was good improvement in the patient's symptoms. Four weeks later the CXR showed marked resolution. ESR titres decreased and there was a significant fall in CRP also. During the next 6 months, steroid dose was tapered gradually and cyclophosphamide was replaced with azathioprin. The patient is presently in remission state.

DISCUSSION: Granulomatosis with polyangiitis (formerly known as Wegener's granulomatosis) is a multisystem disease which is characterised by necrotising granulomatous inflammation of upper and lower respiratory tract, glomerulonephritis and necrotising vasculitis of lungs and a variety of other organs and tissues.

A classification scheme developed by AMERICAN COLLEGE OF RHEMATOLOGY before ANCA antibodies came into practice considered 4 criteria in the definition of GPA:

1. Nasal and oral inflammation.

2. A chest radiography showing presence of nodules fixed inflammation or cavities.

3. Abnormal urinary sediments 4 . Granulomatous inflammation on biopsy specimen.

The presence of 2 or more of these criteria was associated with a diagnostic sensitivity of $88 \%$ and specificity of $92 \%$.

In January 2011, American college of rheumatology (ACR) and American college of nephrology (ACN) and EULAR recommended that the name Wegener's granulomatosis to be changed to GPA to reflect the disease descriptive nomenclature. 
Pathology and Pathogenesis: The immune pathogenesis of GPA is unclear although it suggests an aberrant cell mediated immune response to an exogenous or even endogenous antigen that enters through or resides in upper airways. Chronic nasal carriage of staphylococcus aureus is reported to be associated with higher relapse rate of GPA. More recently it is observed that the complimentary PR3 shows homology with certain Staph. aureus derived peptides which may induce antibodies to PR3.

The histopathological hallmark of GPA is necrotising vasculitis of small arteries and veins together with granuloma formation.

Clinical Maifestations: Upper respiratory tract involvement generally precedes pulmonary or renal involvement. It is the most commonly involved system (90-95\%). Findings are nasal discharge, ulceration, perforation, and sinusitis and subglottic stenosis. The presence of ear involvement varies from 19-45\%. Otological involvement may be in the form of serous otitis media, sensorineural hearing loss, vertigo, facial palsy. Occasionally the tympanic cavity may become filled with granulation tissue and may erode into mastoid cavity as it happened in the present case.

Pulmonary involvement (54-85\%) manifests as cough, haemoptysis, dyspnoea and chest pain. Renal involvement occurs in 51-80\%, Skin lesions in 33-46\%, Eye involvement in 35-52 \%, Neurological manifestations in $20-50 \%$ and Cardiac involvement in $8 \%$.

Radiological Manifestations: Parenchymal abnormalities in a chest $\mathrm{x}$ ray include nodules ranging in size from a few $\mathrm{mm}$ to $10 \mathrm{~cm}$ in diameter. In most cases they are bilateral, $50 \%$ develop cavities.

CT may show presence of more nodules, airspace consolidation or ground glass opacities secondary to pulmonary haemorrhage, wedge like opacities. Pleural effusion, tracheal stenosis, hilar and mediastinal lymphadenopathy are uncommon. In the present case the large size mass like appearance of the lesion with fissural crossing and encasement of lobar bronchus gave rise to suspicion of bronchogenic carcinoma.

Diagnostic Considerations: GPA should be distinguished from other renal syndromes such as Goodpasture's syndrome. Other causes of AAV (ANCA associated vasculitis), infectious causes like mycobacteria and fungi. Malignancies, necrotising sarcoid granulomatosis are the other differential diagnoses to be considered.

The diagnosis of GPA involves demonstration of necrotising granulomas, vasculitis on a tissue biopsy in a patient with compatible clinical features. It is further aided by measurement of serum ANCA levels. C-ANCA (Cytoplasmic anti neutrophilic cytoplasmic antibodies) directed against PR3 is most specific for GPA.

ANCA's are detected with 1) Immunofluorescence 2) ELISA. Immunofluorescence represents qualitative ANCA assay. ELISA provides a target antigen specific characterisation of ANCA i. e anti PR3 and anti MPO and is used to confirm immunofluorescence findings. Combined immunofluorescence and ELISA enhances the sensitivity and specificity of diagnosis of AAV to $96 \%$ and $98.5 \%$ respectively.

Treatment Considerations: Treatment of GPA includes remission induction therapy and remission maintenance therapy. 


\section{CASE REPORT}

Remission Induction Therapy: Standard remission induction therapy for patients with severe disease consists of oral prednisolone and oral cyclophosphamide $2 \mathrm{mg} / \mathrm{kg} /$ day with monitoring of $\mathrm{CBC}$, renal functions. In patients with rapidly progressive disease with alveolar haemorrhage treatment consists of methylprednisolone $1 \mathrm{~g}$ for 3 to 5 days and when it fails plasma exchange should be implemented. Rituximab is an alternative to cyclophosphamide for remission induction therapy.

Remission Maintenance Therapy: Once remission is achieved, prednisolone dose is tapered gradually over the course of 5 to 6 months and cyclophosphamide is switched to either methotrexate or azathioprine and it is continued atleast for 12 months. Use of cotrimoxazole is proved to be beneficial in preventing relapse. The remission rate in GPA varies from 30-93\%. Most morbidity in GPA is currently due to treatment related complications.

CONCLUSIONS: GPA has a wide spectrum of involved sites. Upper airway manifestations are mostly sinonasal and less commonly otological as seen in the present case. This case also has the unusual radiological presentation of a mass like appearance with fissural transgression, mediastinal adenopathy and pleural effusion. GPA should be considered among differential diagnoses in multi systemic diseases. Histological sampling and ANCA assays should be done at the earliest for a definite diagnosis and early institution of treatment so that irreversible damage to the organ can be prevented.

\section{REFERENCES:}

1. Leavitt. RY, Fanci AS, Bloch DA etal. The American college of rheumatology 1990 criteria for the classification of Wegners granulomatosis. Arthritis Rheum. August 1990; 33 (8): 1101-1107.

2. Falk RJ. Gross WL. Gullevin L. et al. Granulomatosis with polyangitis (Wegners) an alternative name for Wegners granulomatosis. Ann Rheum Dis 2011; 70: 704.

3. Pendergraft WF $3^{\text {rd }}$, Preston GA, Shah RR. etal. Auto immunity is triggered by CPR -3 (105-201), a protein complimentary to human autoantigen protinease-3. Nat Med. January 2004; 10 (1); $72-9$.

4. Alfred P. FISHMAN, Daniel H. Stenman, Pulmonary Vasculitis, fouth edition, vol 2, 1449 -1460.

5. Sharma Arpit, Deshmukh shraddha, Dabholkar Jyoti, ENT manifestations of Wegners granulomatosis. Otolaryngologia. Polska 67 (2013); 257-260.

6. Fraser RS, Pare JA. Immunological lung disease, Synopsis of Disease of Chest $3{ }^{\text {rd. }}$ Pennsylvania. Elsevier sunders 2005; page 498 -499.

7. Huck Chin chew, Yew mengchan, Al Jajeh Isaam, Maniko Siynekoh, A patient with hearing loss, mediastinal lymphadenopathy and cavitatory pulmonary nodules Chest 2010; 138 (6): 1500 1514.

8. Moosig F, Lampreclit P, Gross WL, Wegners Granulomatosis, the current view. Clin Rev Allergy Immunol. October 2008; 35 (1-2); 19-21. 


\section{CASE REPORT}

Fig. 1: X-ray chest showing right upper lobe mass like opacity and nodular lesions in mid and lower zones.

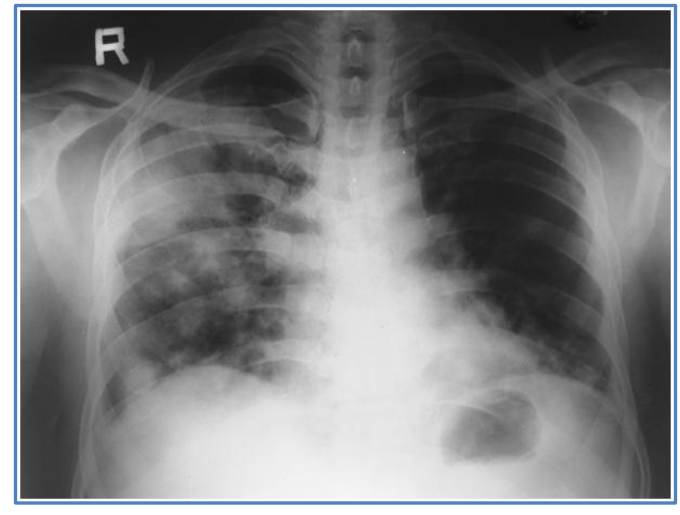

\section{Fig. 1}

Fig. 2: Axial section of CECT thorax showing soft tissue density mass in the right upper lobe and cavitating nodule surrounded by ground glass haziness.

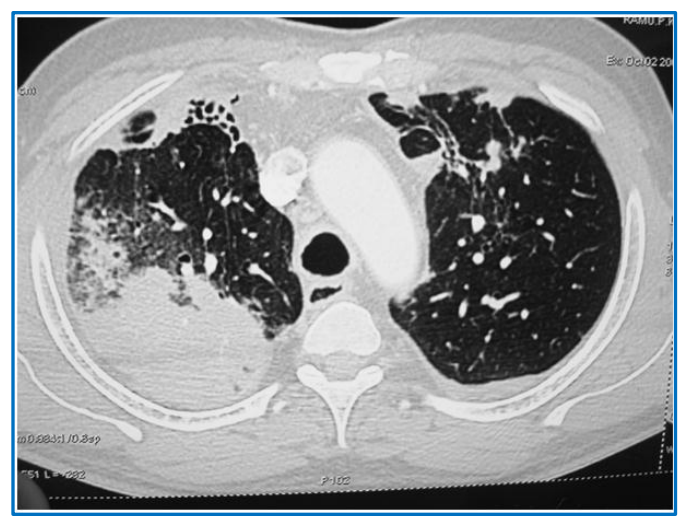

\section{Fig. 2}

Fig 3: CT of temporal bones showing bilateral chronic sclerosing mastoiditis.

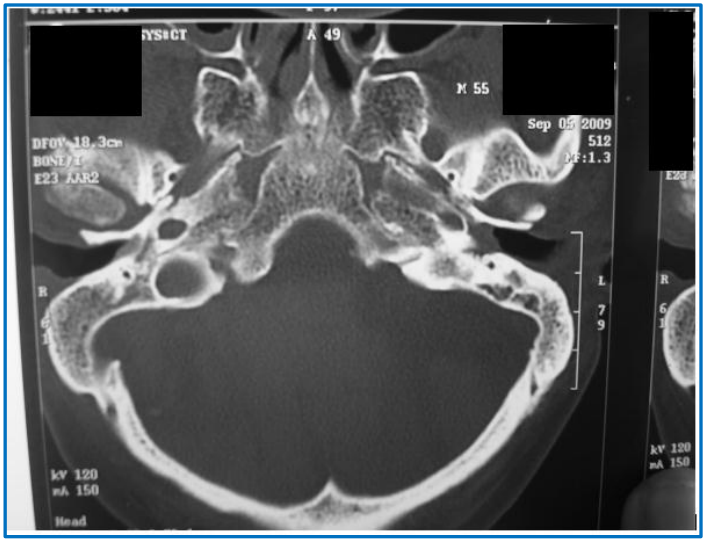

\section{Fig. 3}




\section{AUTHORS: \\ 1. Allena Prem Kumar \\ 2. Namballa Usharani \\ 3. Gayatri Devi Yellapu \\ 4. Gorantla Samba Siva Rao \\ 5. Banavath Durga Prasad Naik}

\section{PARTICULARS OF CONTRIBUTORS:}

1. Professor, Department of Pulmonary Medicine, Andhra Medical College, Visakhapatnam.

2. Associate Professor, Department of Pulmonary Medicine, Andhra Medical College, Visakhapatnam.

3. Assistant Professor, Department of Pulmonary Medicine, Andhra Medical College, Visakhapatnam.

\section{FINANCIAL OR OTHER} COMPETING INTERESTS: None
4. Professor \& HOD, Department of Pulmonary Medicine, Andhra Medical College, Visakhapatnam.

5. Post Gradute, Department of Pulmonary Medicine, Andhra Medical College, Visakhapatnam.

\section{NAME ADDRESS EMAIL ID OF THE CORRESPONDING AUTHOR:}

Dr. Banavath Durga Prasad Naik, Room No. 88, PG Men's Hostel, King George Hospital, Visakhapatnam.

E-mail: durgaprasad900@gmail.com

Date of Submission: 20/03/2015. Date of Peer Review: 21/03/2015. Date of Acceptance: 02/04/2015. Date of Publishing: 16/04/2015. 